\title{
Environmental and genetic control of sexual reproduction in Daphnia
}

\author{
HONG-WEN DENG* \\ Department of Biology, University of Oregon, Eugene, OR 97403, U.S.A.
}

\begin{abstract}
Sexual reproduction in cyclically parthenogenetic Daphnia species is a critical life history component. To investigate the nature of variation of the intensity of sexual reproduction (ISR) in Daphnia, and to measure the relative importance of different aspects of the variation, 30 random clones from a Daphnia pulicaria population were tested in a factorial design involving five photoperiods and two food concentrations. The results revealed that the ISR, indexed by the number of resting eggs produced, is influenced significantly by both the environmental conditions employed (photoperiod, food) and genetic factors (clonal effects within single environments and clone-photo-food interaction across environments). At the population level, a critical photoperiod exists (14 h light/day) for sexual reproduction to be initiated. There are significant differences among the estimates of the genetic variation for the ISR across some environments, signifying that the measurement of genetic variability in one environment has limited value for inferring the magnitude of genetic variability in other environments. There is highly significant genotype-environment $(\mathrm{G} \times \mathrm{E})$ interaction, accounting for about 37.5 per cent of the total variance of the ISR. The results are discussed in the context of the population's adaptation to the ecological surroundings and the maintenance of genetic variability for the ISR in a particular environment.
\end{abstract}

Keywords: cyclical parthenogenesis, Daphnia, genetic (co)variation, genotype-environment interaction, photoperiodism, sexual reproduction.

\section{Introduction}

Water fleas (Daphnia spp) live in either intermittent or seasonally changing permanent environments. In ephemeral environments (typically temporal ponds), populations face the problem of temporary loss of habitable environment as a consequence of pond drying. In permanent environments (usually lakes and reservoirs), populations must cope with seasonal changes in food availability, chemical and physical factors, etc. Extinction of local populations in terms of active living forms, or dramatic reduction of population size and genetic variability, occurs periodically in permanent habitats (Hutchinson, 1967; Lynch, 1984; Threlkeld, 1987; Tessier et al., 1992). As in many other arthropods, diapause has evolved as the means by which water fleas survive during harsh environmental periods (Stross, 1987; Carvalho \& Wolf, 1989).

*Present address: Human Genetics Center, SPH, University of Texas, PO Box 20334 Houston, TX 77225, U.S.A.
Most populations of Daphnia species reproduce by cyclical parthenogenesis. In such populations, the only diapausing form is the sexually produced resting egg, which is wrapped in a modified portion of the carapace. The melanized carapace, together with the two resting eggs inside, is called an ephippium. Each sexual instar produces one ephippium, and each asexual instar releases varying numbers of asexual offspring. Females can switch back and forth between sexual and asexual instars even after sexual reproduction is initiated. In the face of a rapidly disappearing habitat, failure of initiation of sexual reproduction means permanent loss of the genetic material carried by the individual. A slow initiation and/or a slow pace of sexual reproduction will also result in low fitness of individuals, in that genes carried by these individuals will be disproportionately less represented in diapausing 'egg-banks' (Hobaek \& Larsson, 1990; Yampolsky, 1992). On the other hand, during benign periods, allocation by a clone of limited energy to sexual reproduction may slow the rate of asexual reproduction (Hobaek \& Larsson, 1990; Yampolsky, 1992), resulting in slow 
propagation of the genotype prior to sexual reproduction, and hence low fitness of the genotype. Therefore, timing and intensity of sexual reproduction are very important in ensuring the survival of Daphnia populations and in determining the fitness of genotypes in both benign and harsh environments.

To study the initialization of sexual reproduction and the variation for the intensity of sexual reproduction (ISR) in Daphnia, with an aim of understanding its evolution, determining factors need to be identified first. For decades, mechanisms responsible for the variation of sexual reproduction in Daphnia have been subject to intensive studies, the majority of which have concentrated on ecological factors (Berg, 1934; Banta \& Brown, 1939; Stross \& Hill, 1965, 1968; D'Ambramo, 1980; Carvalho \& Hughes, 1983; Stross, 1987; Hobaek \& Larsson, 1990). An array of environmental factors has been tested. Of these, crowding, starvation, chemicals (Banta, 1939), photoperiod and temperature (Stross \& Hill, 1965, 1968), and metabolites (Hobaek \& Larsson, 1990) have been identified to influence sexual reproduction. From these studies, it has been concluded that variation in sexual reproduction in Daphnia is environmental. On the other hand, a few recent studies have revealed that genetic factors may also be involved. Genetic variation for the ISR (indexed by the production of ephippia and/or male offspring) was observed both among clones from different populations (Ferrari \& Hebert, 1982; Korpelainen, 1986, 1987) and among clones from hatching of resting eggs from a single population (Yampolsky, 1992); however, for clones from a single population, genetic variation for the ISR was not detected (Carvalho \& Hughes, 1983).

Although studies on the ecology and genetics of life history traits have merits in isolation, combined studies may provide additional insights. As different genotypes may respond differentially to the same environmental changes (genotype-environment interaction $(\mathrm{G} \times \mathrm{E}))$, and because $\mathrm{G} \times \mathrm{E}$ is well documented in diverse organisms (Service \& Rose, 1985; Gillespie \& Turelli, 1989; Stearns, 1992), the estimation of genetic variation and covariation patterns for important life history traits ought to be carried out across an ecologically relevant range of conditions, instead of under one single condition. There may have been several inadequacies with the previous studies that concentrated on the genetics of sexual reproduction in Daphnia. Evidence for the genetic variation of the ISR comes from the results of the experiments employing clones from different populations (Ferrari \& Hebert, 1982; Carvalho \&
Hughes, 1983; Korpelainen, 1986, 1987), or clones from hatchlings (Yampolsky, 1992) in the lake sediments, which may have contained resting eggs from several previous years (Carvalho \& Wolf, 1989) and/or from other populations by passive migration of resting eggs (Mort \& Wolf, 1986; Crease et al., 1990; De Meester, 1993). Different populations or the same population in different years may face very distinct ecological challenges, so they may have diverged genetically in response. Thus, when assayed side-by-side in one environment, genetic variation for sexual reproduction is not unexpected.

To provide an accurate assessment of environmental and genetic factors controlling variation of sexual reproduction within one population, the present study will address the following questions. (i) Is there any significant genetic variation for the ISR within a single population of Daphnia? (ii) If there is, what might be maintaining this variation? (iii) What is the relative importance of genetic and environmental factors in controlling the ISR?

\section{Materials and methods}

\section{Study population}

The experimental population is located in Lava Lake, a permanent lake in the Oregon Cascades, which has an area of about $40000 \mathrm{~m}^{2}$. In June 1992, the population was sampled at the bottom of the lake from six randomly chosen locations (three near the middle and three near the shore, with each being at least $20 \mathrm{~m}$ apart) throughout the lake. Mature Daphnia individuals $(n=120)$ were isolated into separate $150 \mathrm{~mL}$ beakers, with each beaker containing about $100 \mathrm{~mL}$ lake water. The animals were fed with the green alga Scenedesmus from pure laboratory culture, and maintained in an incubator at $15^{\circ} \mathrm{C}$, with a $12 \mathrm{~L}: 12 \mathrm{D}$ photoperiod before the experiment. In each beaker, the original isolated individual and all subsequent asexually produced offspring were genetically identical (Hebert, 1987), and formed a clone.

\section{Species identification and electrophoresis}

Each clone was subject to morphological identification (Brooks, 1957) and all were placed in the Daphnia pulex-pulicaria group. To determine further the species identity and the breeding system of the population, all 120 clones were assayed by cellulose acetate gel electrophoresis (Hebert \& Beaton, 1989) at ten allozyme loci: $L D H, F U M, M P I, A D, M E$, $M D H, H E X, A P K, P G M, P G I$. The characteristic 
locus $L D H$ was fixed for the $F$ allele, thus identifying the population as D. pulicaria (Hebert et al., 1988, 1989; Lynch et al., 1989). Three loci (FUM, PGI, $P G M$ ) were found to be polymorphic with two, three and three alleles, respectively. $G$-tests (Sokal \& Rohlf, 1981) were performed for these three polymorphic loci against Hardy-Weinberg expectations. None of these tests was significant at the 5 per cent level. The consistency with Hardy-Weinberg expectations indicated that the study population reproduced by cyclical parthenogenesis.

\section{Sex-induction experiment}

The experiment employed a fully crossed threefactorial experimental design (Sokal \& Rohlf, 1981), in which 30 clones were chosen randomly from the 120 established clonal cultures and were used in ten environments of combinations of five photoperiods and two food concentrations. The two environmental variables (photoperiod and food) were chosen on the basis of their ecological relevance and past studies of their roles in controlling sexual reproduction in Daphnia. The five photoperiods used cover those typically experienced by Daphnia populations in temperate regions throughout a year $(8$, $10.5,13,15.5,18 \mathrm{~h}$ of light per day). The two food conditions (100000 and 300000 cells of the alga Scenedesmus per $\mathrm{mL}$ ) approximately mimic the range of food conditions experienced by Daphnia populations (Lynch, 1989; Spitze et al., 1991). The food water preparation protocol was the same as that of Spitze (1991); the water was taken from Lava Lake, to minimize the potential $\mathrm{G} \times \mathrm{E}$ interaction for water chemicals. The experimental temperature was $10^{\circ} \mathrm{C}$, approximating the lake bottom temperature at the time of sampling.

In each environment, one immature female was used to initiate a line for each clone. Upon releasing the first clutch, the clonal line was split into two sublines by isolating a newborn female into each of the two sublines and discarding the mother and all the rest of the newborns. Subsequently, in each subline, first clutch newborns were expelled upon release; and four newborn females from the second clutch were used to initiate the second generation in the subline, i.e. the generation for measurement. The first two generations of acclimatization (one generation in clonal lines and the first generation in sublines) served to minimize the pre-experimental environmental effects and factor any potential maternal effects into the among-subline component of variance in the final ANOVA analysis (Lynch, 1985). Throughout the experiment, the lines and sublines of each clone were kept in $120 \mathrm{~mL}$ food water in $150 \mathrm{~mL}$ beakers. The food water was changed every 3 days throughout the experiment.

In the third generation, the date of maturity for each subline was recorded, with maturity judged by the first appearance of either sexual or asexual reproduction occurring in the beaker. Within the next 20 days after maturiy, the number of released ephippia was counted, and the released sexual offspring were discarded every three days when changing food water. In the experiment, during the 20 days after maturity, each individual had about three sexual instars, or five asexual instars, or some combination of them.

Throughout the experiment, the mortality of clonal lines and sublines was very small (less than 5 per cent throughout the whole experiment, and usually fewer than one (out of 300 or 600) lines or sublines per experimental day). When any line or subline died, it was restarted from backup clonal cultures, which resulted in a balanced data set.

\section{Analysis and results}

\section{Percentage of clones reproducing sexually}

The percentage of clones reproducing sexually was calculated for each of the ten environments. A clone was classified as having reproduced sexually within an environment if any ephippium was released in either one of the two sublines of the clone. In both food conditions, the percentage of sexual clones increased dramatically around the photoperiod of $14 \mathrm{~h}$ of light per day (Fig. 1), demonstrating a critical photoperiod for sexual reproduction to be initiated at the population level. When the photoperiod dropped from $15.5 \mathrm{~h}$ of light per day to $13 \mathrm{~h}$ of light per day, the percentage of sexual clones increased from about 4 per cent to about 70 per cent in the high-food condition, and from 0 per cent to about 50 per cent in the low-food condition.

\section{Mean number of ephippia produced per female (ISR)}

ANOVA analysis The mean number of ephippia produced per female was calculated for each of the two sublines of each clone in each environment. This is used as an index for the ISR. The number of sexual clones in the photoperiods of 15.5 and $18 \mathrm{~h}$ of light per day was very small (less than 5 per cent; Fig. 1), and the variances of the ISR in the four environments-in these two photoperiods (two food conditions within each photoperiod) differed signifi- 
cantly from the other six ( $P$ values of the $F$-test for the homogeneity of variances of the four environments against the other six in the three shorter photoperiods were all smaller than 0.01). Therefore, data from the four environments in these two long photoperiods were excluded from further statistical analyses. The heterogeneity of the variances of the ISR in the six environments of the three short photoperiods $(8,10.5,13 \mathrm{~h}$ light/day) was checked by a Scheffé-Box test; it was not significant at the 5 per cent level. The data were then analysed by a mixedmodel three-way ANOvA for the ISR (Table 1).

Three-way ANOVA was conducted by using PROC GLM (SAS, 1990, chap. 24), specifying clone and its interactions with photoperiod and food as random

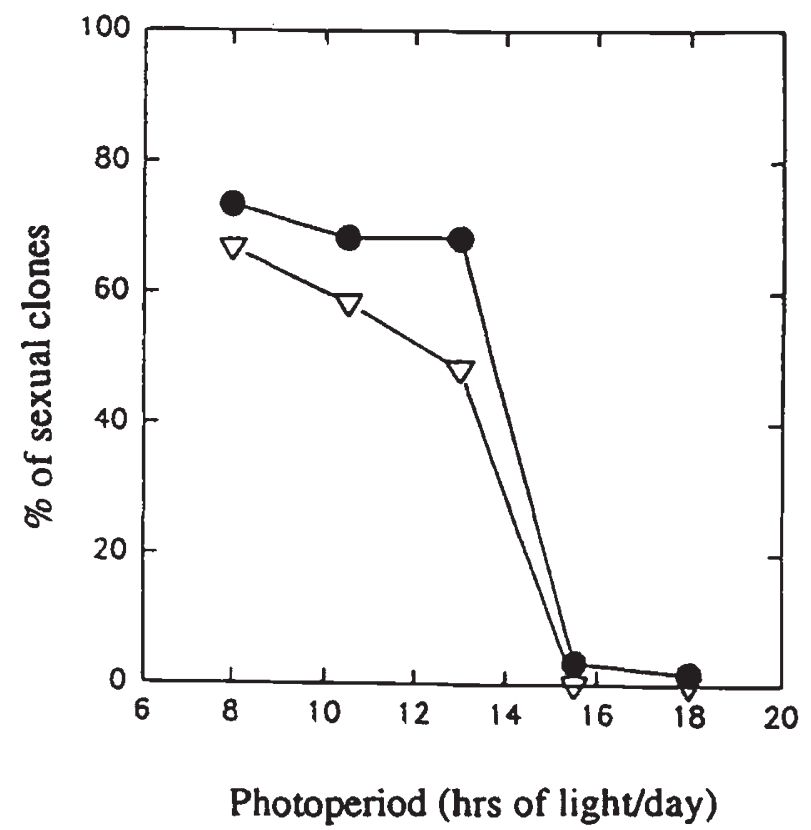

Fig. 1 Percentage of Daphnia clones reproducing sexually in the five experimental photoperiods. The filled circles represent data points for the high-food condition, open triangles for the low-food condition. effects. Photoperiod, food and the photoperiodfood-clone interaction had significant effects on the ISR (Table 1), whereas clone, the photoperiodclone interaction, food-clone interaction, and photoperiod-food interaction did not (Table 1). Hence, in addition to the environmental factors (photoperiod, food), the genetic factor (clone-foodphotoperiod) was also involved in determining the ISR, although a significant role was not detected for the pure genetic effects (clone).

Variance components To estimate the magnitude of the $\mathrm{G} \times \mathrm{E}$ interaction and the importance of genetic factors involved in sexual reproduction, variance components were estimated for the ISR by using PROC VARCOMP (SAS, 1990, chap. 44); (Table 2). Of the total variance, 37.5 per cent was attributable to $\mathrm{G} \times \mathrm{E}$ interaction, 6.7 per cent to pure genetic factors (clone) and 55.8 per cent to the error term. Of the total $\mathrm{G} \times \mathrm{E}$ interaction of 37.5 per cent, 24.5 per cent was attributable to the third-order interaction term, suggesting that the ISR in Daphnia is rather sensitive to particular combinations of environmental factors.

Rank analyses Because of the nonsignificant foodphotoperiod interaction (Table 1), data from the two food conditions in each photoperiod were pooled. The mean number of ephippia produced per individual for each clone in each photoperiod was then calculated. Spearman correlation coefficients were used to quantify the changes of the ranks of the ISR across different pairs of photoperiods (Table 3). Among the three Spearman correlation coefficients, two were low and nonsignificant, and one was significant but low. These Spearman rank correlations indicated rank changes of the ISR among clones across environments.

Table 1 Summary of the three-way ANOVA analysis on the ISR in Drosophila

\begin{tabular}{lrrrr}
\hline Factor & d.f. & \multicolumn{1}{c}{ M.S. } & $F$ value & $P$ value \\
\hline Photoperiod & 2 & 63.17 & 3.99 & 0.024 \\
Food & 1 & 121.34 & 10.24 & 0.003 \\
Clone & 29 & 24.93 & 1.51 & 0.150 \\
Photo $\times$ food & 2 & 8.02 & 0.72 & 0.490 \\
Photo $\times$ clone & 58 & 15.82 & 1.42 & 0.090 \\
Food $\times$ clone & 29 & 11.85 & 1.06 & 0.410 \\
Photo $\times$ food $\times$ clone & 58 & 11.15 & 1.90 & 0.007 \\
Error & 180 & 5.85 & & \\
\hline
\end{tabular}


Genetic variation within environments In the experiment, each clone was represented by two sublines in each environment, which allowed calculation of broad sense heritability $\left(H^{2}\right)$ in each environment and comparison of $H^{2}$ s across environments. The $H^{2}$ is the ratio of $V_{\mathrm{g}}$ (variance component attributable to clone, i.e. total genetic variance) to $V_{\mathrm{p}}$ (total phenotypic variance). The estimates of variance components for one-way ANOVA in each environment were extracted from PROC VARCOMP output (SAS, 1990, chap. 44). The standard error for $H^{2}$ was calculated by using Taylor expansion (Appendix 1). For all environments except the one of high-food and $10.5 \mathrm{~h}$ of light/day, there was significant genetic variation for the ISR indicated by the significant $H^{2}$ (Fig. 2), which was corroborated by the significant pure genetic (clone) effects in the corresponding independent one-way ANOVA analyses. Additionally, there was significant heterogeneity of genetic variation across some environments (Table 4), which indicated that a genetic variation estimate in one environment could be a poor estimate for those in other environments.

Table 2 Variance components and the percentage of total variance associated with clone, clonal interactions with photoperiod and food, and the error variance

\begin{tabular}{lcc}
\hline Variance component & Estimate & Percentage \\
\hline Clone & 0.700 & 6.7 \\
Photo $\times$ clone & 1.172 & \\
Food $\times$ clone & 0.119 & 37.5 \\
Photo $\times$ food $\times$ clone & 2.640 & 55.8 \\
Error & & \\
\hline
\end{tabular}

Estimates of variance components were extracted from SAS output using the VARCOMP procedure, specifying photoperiod and food as fixed effects. The variable is the ISR in Daphnia.
Genetic correlations across environments Sexual reproduction in two environments may be viewed as two distinct characters (Falconer, 1952, 1981; Via \& Lande, 1985, 1987). The methods in Fry (1992) were employed to compute and to conduct significance testing for the genetic correlations for the ISR across different environments. Estimates of genetic correlations across most environments were not significant (Table 5). There were six significantly positive genetic correlations, most of which (except the ones of 0.86 and 0.90 ) were also significantly less than +1 , as tested by the significant $\mathrm{G} \times \mathrm{E}$ interaction in the corresponding two-way mixed-model ANOVA analyses (Yamada, 1962).

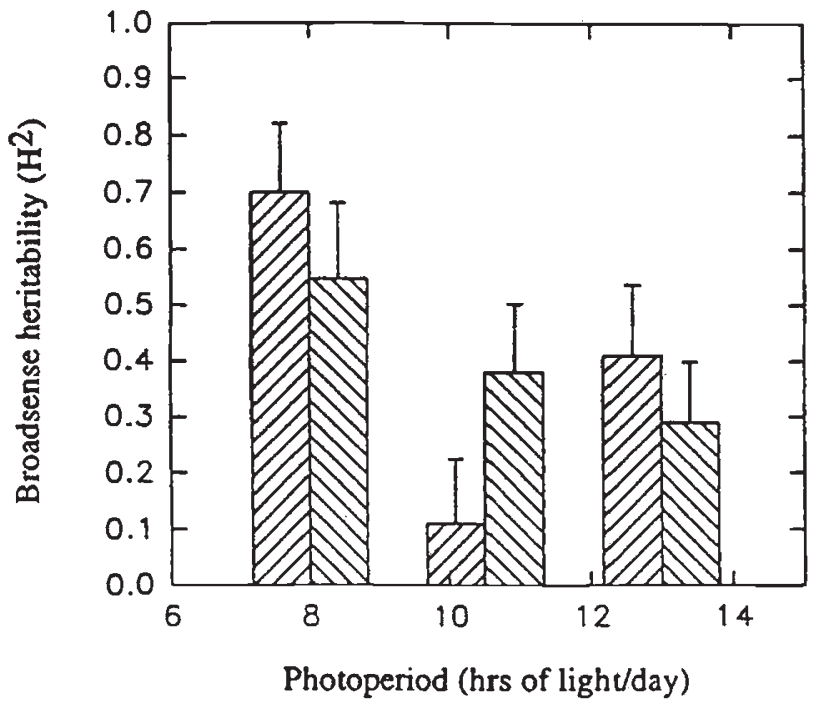

Fig. 2 Broad sense heritabilities $\left(H^{2}\right)$ for the ISR in Daphnia and their associated two standard errors for the two food conditions within each of the three photoperiods $(8,10.5,13 \mathrm{~h}$ of light/day). For each photoperiod, the left bars represent estimates for the high-food condition, the right bars for the low-food condition.

Table 3 Rank correlations between photoperiods for the ISR shown by clones of Daphnia

\begin{tabular}{llcr}
\hline Photoperiod (h of light/day) & 8 & 13.5 & 15 \\
Numerical code for photoperiod & 1 & 2 & 3
\end{tabular}

Spearman rank correlation coefficients for paired photoperiods

\begin{tabular}{lccc}
\hline Paired photoperiods & 1 vs. 2 & 2 vs. 3 & 1 vs. 3 \\
Spearman rank correlation coefficient & 0.246 & $0.411^{*}$ & 0.052 \\
$P$ value & 0.185 & 0.027 & 0.778 \\
\hline
\end{tabular}

${ }^{*} P \leqslant 0.05$. 
Table 4 Differences in broad sense heritability $\left(H^{2}\right)$ of ISR in Daphnia across the six experimental environments

\begin{tabular}{|c|c|c|c|c|c|c|c|}
\hline \multirow{2}{*}{$\begin{array}{l}\text { Photoperiod } \\
\text { (h light/day) }\end{array}$} & \multirow[b]{2}{*}{ Food } & \multicolumn{2}{|c|}{8} & \multicolumn{2}{|c|}{10.5} & \multicolumn{2}{|c|}{13} \\
\hline & & High & Low & High & Low & High & Low \\
\hline \multirow[t]{2}{*}{8} & High & & $\begin{array}{c}0.15 \\
(0.09)\end{array}$ & $\begin{array}{l}0.59^{* *} \\
(0.08)\end{array}$ & $\begin{array}{c}0.32 * * \\
(0.10)\end{array}$ & $\begin{array}{l}0.29^{* *} \\
(0.09)\end{array}$ & $\begin{array}{l}0.41^{* *} \\
(0.10)\end{array}$ \\
\hline & Low & & & $\begin{array}{l}0.44^{* *} \\
(0.09)\end{array}$ & $\begin{array}{c}0.17 \\
(0.09)\end{array}$ & $\begin{array}{c}0.14 \\
(0.10)\end{array}$ & $\begin{array}{l}0.26^{* *} \\
(0.08)\end{array}$ \\
\hline \multirow[t]{2}{*}{10.5} & High & & & & $\begin{array}{r}-0.27^{*} \\
(0.13)\end{array}$ & $\begin{array}{r}-0.30^{*} \\
(0.14)\end{array}$ & $\begin{array}{r}-0.18^{*} \\
(0.08)\end{array}$ \\
\hline & Low & & & & & $\begin{array}{c}-0.03 \\
(0.08)\end{array}$ & $\begin{array}{c}-0.09 \\
(0.09)\end{array}$ \\
\hline \multirow[t]{2}{*}{13} & High & & & & & & $\begin{array}{c}0.12 \\
(0.08)\end{array}$ \\
\hline & Low & & & & & & \\
\hline
\end{tabular}

Shown in each cell is the difference for the two compared environments $\left(H^{2}\right.$ for the environment on the left $-H^{2}$ for the environment on the top). Within parentheses below each difference estimate is the associated one standard error. ${ }^{*} P \leqslant 0.05,{ }^{* *} P \leqslant 0.01$ judged by whether the estimate exceeded $1.96,2.56$ of the associated one standard error.

Table 5 Genetic correlations for the ISR of Daphnia

\begin{tabular}{|c|c|c|c|c|c|c|c|}
\hline \multirow{2}{*}{$\begin{array}{l}\text { Photoperiod } \\
\text { (h light/day) }\end{array}$} & \multirow[b]{2}{*}{ Food } & \multicolumn{2}{|c|}{8} & \multicolumn{2}{|c|}{10.5} & \multicolumn{2}{|c|}{13} \\
\hline & & High & Low & High & Low & High & Low \\
\hline \multirow[t]{2}{*}{8} & High & & $0.54^{* *}$ & -0.13 & $0.51^{* *}$ & -0.09 & -0.23 \\
\hline & Low & & & 0.15 & $0.86^{* *}$ & 0.15 & 0.06 \\
\hline \multirow[t]{2}{*}{10.5} & High & & & & 0.03 & 0.34 & 0.25 \\
\hline & Low & & & & & $0.47^{*}$ & $0.90^{*}$ \\
\hline \multirow[t]{2}{*}{13} & High & & & & & & $0.45^{*}$ \\
\hline & Low & & & & & & \\
\hline
\end{tabular}

${ }^{*} P \leqslant 0.05,{ }^{* *} P \leqslant 0.01$.

\section{Discussion}

The present study showed that within environments and across environments, genetic factors played a role in determining variation and covariation of the ISR within a population of $D$. pulicaria, as manifested by the significant genetic variation (pure genetic effects) within (five of six) environments, and six significant genetic correlations across some environments. Environmental factors, such as photoperiod and food, may play a more important role across the environments tested, as demonstrated by the significant effects for the photoperiod and food, nonsignificant clonal effects in the three-way ANOVA analysis, and the high error term variance (55.8 per cent) in the variance component analysis. Nevertheless, the importance of genetic effects across environments was manifested, in combination with environmental factors, by the significant clonephoto-food interaction in the three-way ANOVA, and by the high percentage of variance attributable to $\mathrm{G} \times \mathrm{E}$ interactions (37.5 per cent).

The high error term variance included contributions from all uncontrolled environmental factors. One of the uncontrolled factors was possibly the density of individuals in each beaker. Although density was controlled at four females to each beaker in the measurement generation, after maturity, asexual offspring were released randomly in time and in number into the beaker, and they were only 
removed every 3 days when changing food and water. This protocol affected the density of each beaker in a more or less random fashion, and thus may have contributed to the large percentage error term variance. Density has been identified as an important ecological factor affecting sexual reproduction in Daphnia (Banta, 1939; Hobaek \& Larsson, 1990).

The results reported here on the photoperiodic response of sexual reproduction in Daphnia are in accord with the previous work of Stross (1987) in $D$. pulex, and Carvalho \& Hughes (1983) in D. magna. They found a critical photoperiod for the initiation of sexual reproduction, but the threshold nature of the critical photoperiods in their studies was more pronounced. The critical photoperiod from the present study (14 h light/day) is about the same as those seen in the previous studies. With some modifications by altitude and latitude, in temperate regions, short photoperiods (less than $14 \mathrm{~h}$ of light/ day) approximately represent the seasons of autumn, winter and early spring, and long photoperiods (longer than $14 \mathrm{~h}$ of light/day) approximately represent the seasons of late spring and summer. As biotic and abiotic environmental factors that control Daphnia population dynamics, temperature, vertebrate and invertebrate predation, food resources, etc. are usually optimum for Daphnia populations' growth in spring and summer in permanent lakes (Hutchinson, 1967; Threlkeld, 1987). Phenology studies have revealed that most permanent lake Daphnia populations in temperate regions crash and initiate sexual reproduction in late summer or early autumn (Threlkeld, 1987 and references within; Stross, 1987 and references within). Thus, the criticial photoperiod for sexual reproduction here coincides with the seasonal timing of sexual reproduction in Daphnia in permanent lakes in temperate regions.

Of note is the high percentage of variance attributable to the third-order interaction term. It is usually assumed that higher-order interactions are of less importance than lower-order ones. This assumption often underlies experimental designs and analyses (Bell, 1990). However, our results and those from Bell (1990) provide no support for this notion. Qualitative support for the present result may come from Stross (1987), who found the combinations of temperature and photoperiod to be critically important in inducing sexual reproduction in Daphnia. Photoperiodic control of sexual reproduction may be completely uncoupled at certain temperatures for some clones (Stross, 1987).

An interesting result arising from the present study is the strong $\mathrm{G} \times \mathrm{E}$ interaction, which accounts for 37.5 per cent of total variance for the ISR across environments. Because strong $\mathrm{G} \times \mathrm{E}$ interaction existed, the nonsignificant pure genetic (clonal) effects in the three-way mixed-model ANOVA analysis did not extend to each single environment. On the contrary, significant pure genetic (clonal) effects were detected in five of six environments analysed. $\mathrm{G} \times \mathrm{E}$ interaction variance is a measure of the degree of genetic variation for reaction norms among genotypes. Significant $\mathrm{G} \times \mathrm{E}$ interaction in ANOVA, as supplemented by the rank and genetic correlation analyses, indicated that genotypes respond differentially to environmental changes. The differential response may be reflected in the scale as well as in the rank of the mean performance among clones, with both contributing to the estimates of $\mathrm{G} \times \mathrm{E}$ interaction. However, it is the latter that is more emphasized in maintenance of genetic variance (e.g. Gupta \& Lewontin, 1982; Via, 1984; Gillespie \& Turelli, 1989; Wade, 1990). The change of the rank among genotypes for fitness traits means that natural selection favours different genotypes in different environments, and it is this kind of $\mathrm{G} \times \mathrm{E}$ interaction that may directly lead to the maintenance of genetic variance within populations (Gupta \& Lewontin, 1982; Gillespie \& Turelli, 1989; Wade, 1990; but see Via \& Lande, 1985, 1987). The rank changes, as shown by the nonsignificant and small Spearman correlation coefficients for the ISR at different photoperiods, indicate that significant $\mathrm{G} \times \mathrm{E}$ interaction in the present study may help to explain the high genetic variability observed within a particular environment. This is because different photoperiods are associated with different times of the year, and the best timing of sexual reproduction, as determined by environmental factors (such as temperature, food resources, and predators, etc.), varies from year to year (Hutchinson, 1967; Threlkeld, 1987; Hairston \& Dillon, 1990). Then different clones, which reproduce sexually most intensively at different photoperiods within a certain seasonal range, are favoured in different years and hence are not absolutely favoured by selection. In another zooplankton species (Diaptomus sanguineus), Hairston \& Dillon (1990) showed that selection for the best timing of diapause fluctuated from year to year. The mean selection intensity over a decade for this fluctuating selection was essentially zero, which was invoked to explain the high genetic variability observed in their study.

In the present study, significant heterogeneity of genetic variances across environments and the co-ocurring of positive and negative genetic correlations across environments point out the advantage of 
combined study of genetics and ecology. Because of labour constraints, estimates of genetic parameters (genetic variation and covariation) have usually been obtained for only one environment, often a laboratory environment. If significant $\mathrm{G} \times \mathrm{E}$ interaction exists, such estimates may have limited value in inferring selection and predicting evolution of a population in its natural heterogeneous environments. Inferring selection in the past and predicting evolution in the future for quantitative traits (Lande, 1979; Lande \& Arnold, 1983) require approximate constancy of the genetic variance-covariance structure of the population over time and space, when and where selection and evolution occur. In the absence of complete ecological and genetic information, the task of evolutionary inference and prediction may be compromised by the presence of significant $\mathrm{G} \times \mathrm{E}$.

\section{Acknowledgements}

I thank Z. Banks, S. Sterry, Q. Tian, S. K. Lee and S. L. Yu for laboratory assistance, N. Lehman, W. Bradshaw, M. Lynch, M. Fugate, M. Pfrender, K. Spitze, S. Via and R. Lande for comments on early versions of the manuscript. I am indebted to $\mathrm{W}$. Bradshaw and $\mathrm{M}$. Lynch for encouragement, suggestions and discussions throughout the initiation, execution and analyses of the experiment. The work was supported by NSF grant BSR 89-11038 to Mike Lynch.

\section{References}

BANTA, A. M. 1939. General characterization of Cladocera. In: Banta, A. M. (ed.) Studies on the Physiology, Genetics and Evolution of Some Cladocera, pp. 2-12. Carnegie Institute, Washington, DC.

BANTA, A. M. AND BROWN, L. A. 1939. Control of male and sexual egg reproduction. In: Banta, A. M. (ed.) Studies on the Physiology, Genetics and Evolution of Some Cladocera, pp. 106-129. Carnegie Institute, Washington DC.

BELL, G. 1990. The ecology and genetics of fitness in Chlamydomonas. I. Genotype-by-environment interaction among pure strains. Proc. R. Soc. B, 240, 295-321.

BERG, K. 1934. Cyclic reproduction, sex determination and depression in the Cladocera. Biol. Rev., 9, 139-174.

Brooks, J. L. 1957. The Systematics of North American Daphnia. The Academy, New Haven, CT.

BUlmer, M. G. 1980. The Mathematical Theory of Quantitative Genetics. Oxford University Press, New York.

CARVAlHo, G. R. AND hUGHes, R. H. 1983. The effects of food availability, female culture density, and photoperiod on ephippia production in Daphnia magna Straus (Crustacea: Cladocera). Freshwater Biol., 13, 37-46.
CARVALHO, G. R. AND WOLF, H. G. 1989. Resting eggs of lake-Daphnia. I. Distribution, abundance and hatching of eggs collected from various depths in lake sediments. Freshwater Biol., 2, 459-470.

CREASE, T. J., LYNCH, M. AND SPITZE, K. 1990. Hierarchical analysis of population genetic variation in mitochondrial and nuclear genes of Daphnia pulex. Mol. Biol. Evol., 7, 444-485.

D'AMBRAMO, L. R. 1980. Ingestion rate decrease as the stimulus for sexuality in populations of Moina macrocopa. Limnol. Oceanogr., 25, 422-429.

DE MEESTER, L. 1993. Inbreeding and outbreeding depression in Daphnia. Oecologia, 96, 80-84.

FALCONER, D. S. 1952. The problem of environment and selection. Am. Nat., 86, 293-298.

FALCONER, D. S. 1981. Introduction to Quantitative Genetics, 2nd edn. Longman, New York.

FERRARI, D. C. AND HEBERT, P. D. N. 1982. The induction of sexual reproduction in Daphnia magna: genetic differences between arctic and temperate populations. Can. J. Zool., 60, 2143-2148.

FRY, J. D. 1992. The mixed-model analysis of variance applied to quantitative genetics: biological meaning of parameters. Evolution, 46, 540-550.

GILLESPIE, J. H. AND TURELLI, M. 1989. Genotype-environmental interactions and maintenance of polygenic variation. Genetics, 121, 129-138.

GUPTA, A. P. AND LEWONTIN, R. C. 1982. A study of reaction norms in natural populations of Drosophila pseudoobscura. Evolution, 36, 934-938.

HAIRSTON, N. G. AND DILLON, T. A. 1990. Fluctuating selection and response in a population of freshwater copepods. Evolution, 44, 1796- 1805

HeBerT, P. D. N. 1987. Genetics of Daphnia. In: Peters, R. H. and de Bernardi, R. (eds) Daphnia. Memorie Istituto Italiano Di Idrobiologia, 45, 439-460.

HEBERT, P. D. N. AND BEATON, M. J. 1989. Methodologies for Allozyme Analysis Using Cellulose Acetate Electrophoresis: a Practical Handbook. Helena Laboratories, Beaumont, TX.

HEBERT, P. D. N., WARD, R. D. AND WEIDER, L. J. 1988. Clonal-diversity patterns and breeding-system variation in Daphnia pulex, an asexual-sexual complex. Evolution, 42, 147-159.

HEBERT, P. D. N., BEATON, M. J. AND SCHWARTZ, s. S. 1989. Polyphyletic origin of asexuality in Daphnia pulex. I. Breeding system variation and levels of clonal diversity. Evolution, 43, 1004-1015.

HOBAEK, A. AND LARSSON, P. 1990. Sex determination in Daphnia magna. Ecology, 71, 2255-2268.

hutchinson, G. E. 1967. A Treatise on Limnology, vol. 2. John Wiley \& Sons, New York.

KORPELAINEN, H. 1986. Reproductive strategies of Daphnia magna genotypes. Hereditas, 106, 181-188.

KORPELAINEN, H. 1987. The effects of temperature and photoperiod on life-history parameters of Daphnia magna (Crustacea: Cladocera). Freshwater Biol., 16, 615-620.

LANDE, R. 1979. Quantitative genetic analysis of multi-

(C) The Genetical Society of Great Britain, Heredity, 76, 449-458. 
variate evolution applied to brain:body size allometry. Evolution, 33, 402-416.

LANDE, R. AND ARNOLD, s. J. 1983. The measurement of selection on correlated characters. Evolution, 37, $1210-1226$.

LYNCH, M. 1984. The limits of life history evolution in Daphnia. Evolution, 38, 465-482.

LYNCH, M. 1985. Spontaneous mutations for life history characters in an obligate parthenogen. Evolution, 39, 804-818.

LYNCH, M. 1989. The life history consequences of resource depression in Daphnia pulex. Ecology, 70, 246-256.

LYNCH, M., SPITZE, K. AND CREASE, T. 1989. The distribution of life-history variation in the Daphnia pulex complex. Evolution, 43, 1724-1736.

MORT, M. A. AND WOLF, H. G. 1986. The genetic structure of large lake Daphnia populations. Evolution, 40, $756-766$.

SAS INSTITUTE. 1990. SAS/STAT User's Guide, vols 1 and 2, Version 6, 4th edn. Cary, NC.

SEARLE, S. R. 1971. Linear Models. Wiley, New York.

SERVICE, P. M. AND ROSE, M. R. 1985. Genetic covariation among life-history components: the effects of novel environments. Evolution, 39, 943-945.

SOKAL, R. R. AND ROHLF, R. J. 1981. Biometry, 2nd edn. Freeman, New York.

STEARNS, s. C. 1992. The Evolution of Life Histories. Oxford University Press, New York.

SPITZE, K. 1991. Chaoborus predation and life-history evolution in Daphnia pulex: temporal pattern of population diversity, fitness, and mean life history. Evolution, 45, 82-92.

SPITZE, K., BURNSON, J. AND LYNCH, M. 1991. The covariance structure of life-history characters in Daphnia pulex. Evolution, 45, 1081-1090.

STROss, G. 1987. Photoperiodism and phased growth in
Daphnia populations: coactions in perspective. In: Peters, R. H. and de Bernardi, R. (eds) Daphnia. Memorie Istituto Italiano Di Idrobiologia, 45, 367-388.

STROSS, P. M. AND HILL, J. C. 1965. Diapause induction in Daphnia requires two stimuli. Science, 150, 1462-1464.

STRosS, P. M. AND HILl, J. C. 1968. Photoperiod control of winter diapause in the freshwater crustacean, Daphnia. Biol. Bull., 134, 176-198.

TESSIER, A. J., YOUNG, A. AND LEIBOLD, M. 1992. Population dynamics and body size selection in Daphnia. Limnol. Oceanogr., 37, 1-14.

THRELKELD, s. T. 1987. Daphnia population fluctuations: patterns and mechanisms. In: Peters, R. H. and de Bernardi, R. (eds) Daphnia. Memorie Istituto Italiano Di Idrobiologia, 45, 367-388.

VIA, s. 1984. The quantitative genetics of polyphagy in an insect herbivore. I. Genotype-environment interaction in larval performance on different host plant species. Evolution, 38, 881-895.

VIA, S. AND LANDE, R. 1985. Genotype-environment interaction and the evolution of phenotypic plasticity. Evolution, 39, 505-522.

VIA, S. AND LANDE, R. 1987. Evolution of genetic variability in a spatially heterogeneous environment: effects of genotype-environment interaction. Genet. Res., 49, $147-156$.

WADE, M. J. 1990. Genotype-environment interaction for climate and competition in a natural population of flour beetles, Tribolium castaneum. Evolution, 44, 2004-2011.

YAMADA, Y. 1962. Genotype-environment interaction and genetic variation of the same trait under different environments. Jap. J. Genet., 37, 498-509.

YAMPOLSKY, L. YU. 1992. Genetic variation in the sexual reproduction rate within a population of a cyclical parthenogen, Daphnia magna. Evolution, 46, 833-837.

\section{Appendix 1}

Calculation of broad sense heritability $\left(H^{2}\right)$ and its standard error by performing one-way ANOVA involving $N$ independent clone families, the $i$ th of which contains $n_{i}$ individuals. The linear model to be analysed is $Z_{i j}=\mu+b_{i}+w_{i j}$, where $Z_{i j}$ is the phenotypic value of the $j$ th individual of the $i$ th clone, $b_{i}$ is the genotypic value of the $i$ th clone, and $w_{i j}$ is the residual error resulting from environmental effects. The total sample size is $T=\Sigma n_{i}$, and $n_{\mathrm{o}}=\left(T-\Sigma n_{i}^{2} / T\right) /(N-1)$, which reduces to $n$ with balanced data.

Source of variance

Between clones

Within clones

Total

$$
\text { d.f. }
$$

$\mathrm{N}-1$

$\mathrm{T}-\mathrm{N}$

$\mathrm{T}-1$
SS

$\mathrm{SS}_{\mathrm{b}}=\Sigma n_{i}\left(z_{i}-z\right)^{2}$

$\mathrm{SS}_{\mathrm{w}}=\Sigma \Sigma\left(z_{i j}-z_{i}\right)^{2}$

$\mathrm{SS}_{\mathrm{T}}=\Sigma \Sigma\left(z_{i j}-z\right)^{2}$
MS

$$
\begin{aligned}
& \mathrm{MS}_{\mathrm{b}}=\mathrm{SS}_{\mathrm{b}} /(N-1) \\
& \mathrm{MS}_{\mathrm{w}}=\mathrm{SS}_{\mathrm{w}} /(T-N) \\
& \mathrm{MS}_{\mathrm{z}}=\mathrm{SS}_{\mathrm{T}} /(T-1)
\end{aligned}
$$

Expected MS

$\sigma_{\mathrm{e}}^{2}+n_{\mathrm{o}} \sigma_{\mathrm{G}}^{2}$
$\sigma_{\mathrm{e}}^{2}$
$\sigma_{\mathrm{z}}^{2}$

Set the mean squares equal to their expectation, and solve the system of linear equations:

$\sigma_{\mathrm{e}}^{2}=\mathrm{MS}_{\mathrm{w}}$, 
$\sigma_{\mathrm{G}}^{2}=\left(\mathrm{MS}_{\mathrm{b}}-\mathrm{MS}_{\mathrm{w}}\right) / n_{\mathrm{o}}$.

Under the assumption of normality, the mean squares are independent and have variances that are estimated by:

$\operatorname{Var}\left(\mathrm{MS}_{i}\right)=2\left(\mathrm{MS}_{i}\right)^{2} /\left(d f_{i}+2\right)$

where $i=b, w$ (Bulmer, 1980, chap. 6).

Thus by taking variances:

$\operatorname{Var}\left(\sigma_{\mathrm{e}}^{2}\right)=\operatorname{Var}\left(\mathrm{MS}_{\mathrm{w}}\right)$,

$\operatorname{Var}\left(\sigma_{\mathrm{G}}^{2}\right)=\left[\operatorname{Var}\left(\mathrm{MS}_{\mathrm{b}}\right)+\operatorname{Var}\left(\mathrm{MS}_{\mathrm{w}}\right)\right] / n_{\mathrm{o}}^{2}$

Broad sense heritability is defined as:

$H^{2}=\sigma_{\mathrm{G}}^{2} / \sigma_{\mathrm{z}}^{2}$

where $\sigma_{\mathrm{z}}^{2}=\sigma_{\mathrm{e}}^{2}+\sigma_{\mathrm{G}}^{2}$. The sampling variance of $\sigma_{\mathrm{z}}^{2}$ is:

$\operatorname{Var}\left(\sigma_{\mathrm{z}}^{2}\right)=\operatorname{Var}\left(\sigma_{\mathrm{e}}^{2}\right)+\operatorname{Var}\left(\sigma_{\mathrm{G}}^{2}\right)+2 \operatorname{cov}\left(\sigma_{\mathrm{G}}^{2}, \sigma_{\mathrm{e}}^{2}\right)$

where $\operatorname{cov}\left(\sigma_{\mathrm{G}}^{2}, \sigma_{\mathrm{e}}^{2}\right)=-T(N-1) \operatorname{Var}\left(\sigma_{\mathrm{e}}^{2}\right) /\left(N^{2}-S_{2}\right)$ and $S_{2}=\Sigma n_{i}^{2}($ Searle, 1971).

By use of Taylor expansion (Bulmer, 1980,chap. 6):

$\operatorname{Var}\left(H^{2}\right)=\left(\sigma_{\mathrm{G}}^{2} / \sigma_{z}^{2}\right)^{2}\left[\operatorname{Var}\left(\sigma_{\mathrm{G}}^{2}\right) / \sigma_{\mathrm{G}}^{4}-2 \operatorname{cov}\left(\sigma_{\mathrm{G}}^{2}, \sigma_{z}^{2}\right) / \sigma_{\mathrm{G}}^{2} \sigma_{\mathrm{z}}^{2}+\operatorname{Var}\left(\sigma_{\mathrm{G}}^{2}\right) / \sigma_{\mathrm{z}}^{4}\right]$. 